\title{
Evaluación del componente forestal de la Reserva Biológica Lomas Barbudal
}

\section{Evaluation of the Forest Component of Lomas Barbudal Biological Reserve}

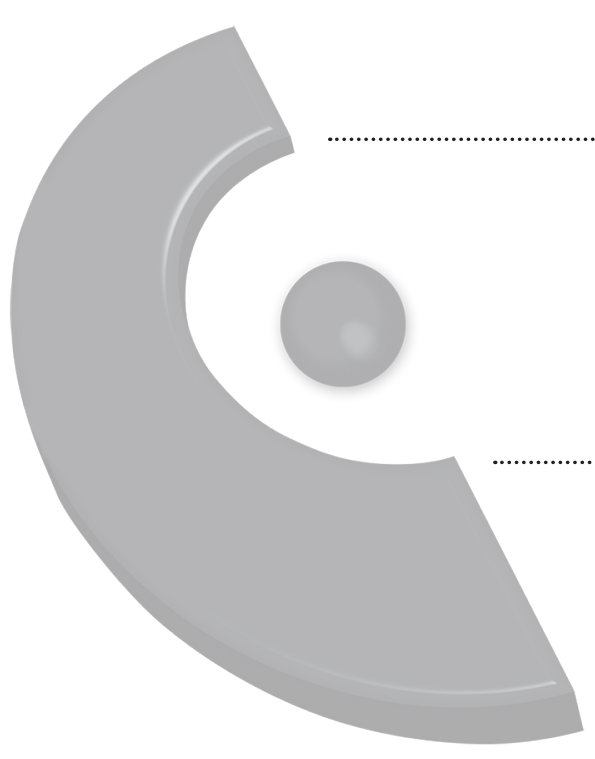

\author{
William Gómez Solís ${ }^{1}$ \\ Universidad Nacional \\ Heredia, Costa Rica \\ willgs88@gmail.com
}

Recibido 07/02/2017 Aceptado 24/05/2017

Resumen: La Reserva Biológica Lomas Barbudal (RBLB) se encuentra ubicada en San Ramón de Bagaces, Guanacaste y cuenta con una extensión de 2645,71 hectáreas. El objetivo de este trabajo es analizar la situación del componente arbóreo en esta reserva, con el propósito de determinar en qué condición se encuentran los recursos forestales existentes por medio de un inventario forestal, el cual incluye servicios ecosistémicos, índices de diversidad, importancia ecológica y caracterización del bosque. El resultado demostró que las especies con mayor índice de valor de importancia (IVI) fueron: Sweitenia macrophylla, Bursera simarouba, Astronium graveolens y Pseudobombax septenatum. Por otra parte, la mayoría de individuos registrados presentó buena forma, lo que demuestra el alto potencial semillero de esta reserva, así como su importancia en cuanto a conectividad con otras áreas protegidas.

1 Tesis sometida a evaluación para obtener el grado de Licenciatura en Manejo Forestal, de Ingeniería en Ciencias Forestales, Escuela de Ciencias Ambientales, Universidad Nacional, Heredia, Costa Rica. 
Revista Universidad en DiÁlogo • Vol. 7, N. ${ }^{\circ}$ 1, Enero-Junio 2017, pp. 101-122

ISSN 2215-2849 • EISSN: 2215-4752

DOI: http://dx.doi.org/10.15359/udre.7-1.6

Palabras claves: caracterización, servicios ecosistémicos, índices de diversidad, importancia ecológica, áreas protegidas.

\begin{abstract}
Lomas Barbudal Biological Reserve (LBBR) is located in San Ramon, Bagaces, Guanacaste, Costa Rica, and has an area of 6,537.69 acres. This research aims to analyze the situation of the arboreal component in the Biological Reserve in order to assess the conditions of the forest resources. These conditions are determined through a forest inventory, which includes ecosystem services, diversity indices, ecological importance, and characterization of the forest. Results showed that species with higher Value Index Importance (V.I.I.) were: Sweitenia macrophylla, Bursera simarouba, Astronium graveolens, and Pseudobombax septenatum. On the other hand, most of the registered species had a good condition, demonstrating the high potential of seeds in this Reserve and its importance of connectivity with other protected areas.
\end{abstract}

Keywords: characterization, ecosystem services, diversity indices, ecological importance, protected areas.

\title{
Introducción
}

A medida que aumenta la población mundial, así como la industria presenta mayor desarrollo agropecuario y tecnológico, resulta cada vez menor la superficie de la Tierra libre de interferencias humanas, pues sus actividades han modificado el ambiente al punto de que los patrones estructurales más comunes son paisajes de asentamientos humanos, terrenos agrícolas y fragmentos dispersos de ecosistemas naturales (Murrieta, 2006). Como consecuencia hubo un considerable descenso y fragmentación en los hábitats naturales. En Guanacaste, por ejemplo, los bosques se vieron afectados en vista de que era necesario darles paso a actividades como la agricultura y la ganadería. Esta realidad afectó fuertemente los bosques secos, por lo cual, según González (2002, p. 147), se les llegó a considerar "el ecosistema en mayor peligro de extinción" en Costa Rica.

El bosque tropical seco es uno de los ambientes más vulnerables del país, además de uno de los más escasos en Centroamérica. Una de sus principales características es que permanece seco por más de noventa días al año, ubicado ya sea sobre topografía plana o plana ondulada o en cerros o colinas irregulares con suelos de tipo latisol profundo, bien drenados; es decir, que no se inundan. Esto implica que todas las plantas y los animales que habitan allí han desarrollado las adaptaciones evolutivas apropiadas para subsistir en condiciones de disponibilidad de nutrientes y de agua 
muchísimo menor que en el bosque lluvioso (Alvarado, 15 de abril, 2013, comunicación personal), lo que obliga a la mayoría de las plantas a perder sus hojas durante la sequía. Además, enfrentan incendios provocados precisamente durante la época del año más seca y cálida.

Por estas condiciones vegetativas, la Reserva Biológica Lomas Barbudal conserva muestras muy valiosas de flora y fauna de la sabana seca arbolada de bajura, del bosque ripario de tierras bajas y del bosque caducifolio de tierras bajas, las cuales en el pasado cubrieron extensas áreas de la provincia de Guanacaste. Otro de los aspectos relevantes por considerar es que el tipo de suelo no presenta condiciones para un desarrollo agropecuario sostenido, pero desde el punto de vista de la investigación y del turismo, el área presenta una fauna entomológica única y contiene sabanas secas que no se encuentran protegidas en otra unidad de conservación (Alvarado, 15 de abril, 2013, comunicación personal).

Además, el área posee una extraordinaria biodiversidad en la que destacan grupos como aves, insectos, mamíferos y plantas. En este último, conviene resaltar las especies en peligro de extinción, tal como el caoba (Swetenia macrophylla) y algunas con distribución muy restringida, como el árbol "balas de cañón" (Couropita nicaraguensis), entre otras (Serrano, 2012).

Debido a lo anterior, determinar el estado de las diferentes poblaciones arbóreas presentes en la Reserva Biológica Lomas Barbudal es una tarea que permitirá establecer una base sobre el valor ecológico/económico de los diferentes servicios ecosistémicos presentes en la zona, según los parámetros de valor de las diferentes especies y el potencial del banco genético del área, entre otros. A medida que se incrementan las presiones sobre los recursos naturales en el mundo, se reconoce que las herramientas ambientales y sociales que guían el desarrollo económico son, ante todo, de carácter reactivo y tienen un papel marginal. Los resultados negativos de esta situación son evidentes: pérdida de servicios ecosistémicos críticos - de los cuales depende la sociedad humana-y extinción acelerada de especies (Andrade, 2007). Esto, junto a la creciente probabilidad de cambio climático severo, hace necesaria la adopción de enfoques proactivos hacia el desarrollo sostenible.

\section{Materiales y métodos}

Para caracterizar los bosques se debe partir de la interrogante ¿cuánto vale la naturaleza? En vista de la necesidad de asignarle un valor a la biodiversidad y los beneficios que brinda, surge la duda anterior al introducir la naturaleza 
Revista Universidad en DiÁlogo • Vol. 7, N. ${ }^{\circ}$ 1, Enero-Junio 2017, pp. 101-122

ISSN 2215-2849 • EISSN: 2215-4752

DOI: http://dx.doi.org/10.15359/udre.7-1.6

dentro de los mercados y asignarle valores económicos. La caracterización de los bosques nace del requerimiento de evaluar la biodiversidad vista como un ecosistema, lo que resulta en la clasificación de las comunidades vegetales, además de constituir el primer paso para entender la estructura y dinámica de estos, por lo que reconocer e interpretar su distribución espacial es fundamental para conservar su gran diversidad (Murrieta, 2006). La información básica sobre los ecosistemas protegidos constituye una herramienta importante en la implementación de medidas adecuadas para su manejo y conservación efectiva en el largo plazo, especialmente de aéreas reducidas o fragmentadas (Cascante y Estrada, 2000).

Por ello, se realizó un mapeo de las diferentes formaciones vegetales presentes en la reserva con el apoyo de imágenes aéreas y visitas de campo, cuyo propósito fue contar con una base para llevar a cabo el análisis del potencial de conectividad del área y definir las formaciones vegetativas dentro del área silvestre protegida. Lo anterior se efectuó desde el criterio de tamaño de fragmentación, el cual, por medio de imágenes aéreas y ensayos de campo, determinó el número de parcelas de muestreo dentro de cada estrato y su respectiva distribución. Se procedió a realizar entonces un análisis del paisaje.

El mapeo del área de estudio se orienta a definir la cantidad de parcelas de muestreo y el lugar que estas ocupan en cada tipo de bosque o estrato, al determinar su adecuado distanciamiento y, también, al evitar condiciones distintas en su respectiva distribución, conforme el área de cada estrato. De este modo, su ubicación será de forma sistemática.

Durante todo el proceso se implementó el protocolo definido por CATIE (2002), el cual ha sido utilizado en otros estudios similares, tales como los de Doblado (2011) y Ramos (2004), además de aplicar el conocimiento en experiencias propias en inventarios de bosques. Para el muestreo de la vegetación, se establecieron parcelas temporales en forma circular de $12.61 \mathrm{~m}$ de radio, con un área de $500 \mathrm{~m}^{2}$ cada una, separadas entre sí al menos con 400 $\mathrm{m}$ de distancia. Esto debido a que se realizará un diagnóstico de la vegetación de la RB Lomas Barbudal, por lo que en los resultados estadísticos se habla de intensidad de muestreo y no de error de muestreo. Además, con este método se pretende abarcar toda la diversidad ecológica presente en el sitio.

Los parámetros a emplear para medir dentro de las parcelas son la especie de todos los individuos y el diámetro a la altura del pecho (DAP) a 1.30 $\mathrm{m}$ y de las especies mayores, a $10 \mathrm{~cm}$. A partir de estas estimaciones se determinó la valoración ecosistémica del componente forestal, el estado 
de las formaciones vegetales presentes y sus respectivos índices de diversidad. Según los datos generados, se evaluarán las poblaciones más representativas de árboles, pues también estos datos serán la base para la determinación del potencial de conectividad del área de estudio, por medio de mapeos y de la evaluación de las especies presentes en la zona.

Los índices de diversidad se calculan generalmente con base en la riqueza de especies y la uniformidad de su contribución a la comunidad; es decir, en qué medida estas abundan por igual (Finegan et al., 2001 y Magurran, 1989, citados por Ramos, 2004). Una alta uniformidad significa que las especies son iguales o virtualmente iguales en abundancia, se equipara con una alta diversidad. Esto con el fin de medir la incertidumbre de la siguiente pregunta: ¿Cuán difícil sería predecir correctamente la especie del siguiente individuo capturado?

La riqueza de especies se entiende como el número total de especies por unidad de área, y la diversidad como el número de especies respecto al tamaño (abundancia) de la población de cada especie (Murrieta, 2006). Por otro lado, la diversidad de ecosistemas boscosos se entiende como el número, variedad y arreglo espacial de los tipos de bosques a una escala dada, de donde se expresa que la diversidad $\beta$ es el grado en el cual las comunidades difieren unas de otras a lo largo de gradientes ambientales (Ramos, 2004).

La importancia ecológica se evalúa a través del índice de valor de importancia (IVI) propuesto por Curtis y McIntosh (1950). Este índice incluye parámetros tales como la abundancia, dominancia y frecuencia de cada especie y proporciona el reconocimiento de la importancia ecológica de una especie en el seno de la comunidad (Lamprecht, 1990, citado por CATIE, 2002).

La abundancia relativa es el porcentaje que representa la especie considerada con respecto al total de árboles de la parcela de estudio, es una estimación de la cobertura de cada especie con respecto al total, expresada como proyección horizontal de copas. Sin embargo, resulta complicado medir dicha proyección en un bosque tropical debido a su compleja estructura. Por ello, varios estudios proponen que se utilice el área basal a fin de sustituir la proyección de copas. La dominancia relativa sería el porcentaje de área basal de cada especie con respecto al total. La frecuencia relativa corresponde al patrón de ocurrencia de los individuos de una especie dentro de una comunidad y determina la regularidad de la distribución horizontal de cada una de ellas, lo que indica la diversidad o complejidad del bosque. 
Revista Universidad en DiÁlogo • Vol. 7, N. ${ }^{\circ}$ 1, Enero-Junio 2017, pp. 101-122

ISSN 2215-2849 • EISSN: 2215-4752

DOI: http://dx.doi.org/10.15359/udre.7-1.6

Además se estimó el índice de similitud de Simpson, el cual evalúa el grado en el que dos comunidades se parecen. Otro parámetro evaluado fue el volumen en metros cúbicos de los individuos registrados, a partir de un modelo de estimación con los elementos diámetro y altura; también se estimó el stock de carbono en la biomasa viva por medio de una ecuación alométrica desarrollada por Chave y colaboradores (2005) para el bosque seco. Como parte del análisis de las poblaciones arbóreas presentes en RB Lomas Barbudal, también se analizó la forma de los árboles muestreados, por medio de una clasificación de la forma de copa y fuste, el grado de iluminación que reciben y la presencia de enredaderas y bejucos tanto en copa como fuste.

\section{Resultados}

La Reserva Biológica Lomas Barbudal posee recursos de biodiversidad en flora y fauna, además de algunas fuentes hídricas como el río Cabuyo, varias quebradas y nacientes naturales que, incluso en la estación seca, se mantienen con abundante agua (Porta, 2011). Estos constituyen recursos valiosos presentes en pocas regiones del país, debido a que la RBLB protege la vegetación típica del bosque tropical seco: los bosques semideciduo y deciduo, el bosque semideciduo de bajura y el bosque siempre verde estacional. Esto se detallará a continuación:

-Bosques semideciduo y deciduo: Ambas categorías se tomaron juntas dado que comparten muchas características en común, tales como que provienen de suelos de origen volcánico, con una topografía que pasa de lo plano a cerros o colinas con pendientes irregulares. Secos por más de noventa días al año, por efecto de una estación seca definida y prolongada. Las especies dominantes pierden su follaje en algún grado, como pueden hacerlo también los estratos dominados y el sotobosque. Se encuentran en zonas expuestas y "cejas de montaña" con vegetación siempre verde, principalmente en las alturas menores y sobre cursos de agua, la cual se sitúa sobre formas estructurales, con calizas y rocas metamórficas. Existe, además, gran cantidad de especies asociadas al ecosistema y las especies más sobresalientes de vegetación son: Guazuma ulmifolia (guácimo), Luehea candida (guácimo molenillo), Spondias mombin (jobo), Bursera simaruba (indio desnudo), Enterolobium cyclocarpum (guanacaste), Bombacopsis quinatum (pochote), Coclospermum vitifolium (poro poro), Acacia spp., Pithecellobium saman (cenízaro), Gliricidia sepium (madero negro), Astronium graveolens (ron-ron), Licania arborea (alcornoque), Caesalpinea eriostachys (saíno), Tabebuia spp., entre otras (Porta, 2011). 
-Bosque semideciduo de bajura: Ocupa la vertiente pacífica de la cordillera volcánica a lo largo del país, entre los 600 y 2000 metros de altitud y los valles intermontanos interiores; además de sabanas arboladas cubiertas de pastos y árboles diseminados y matorrales de Acasia spp. (Porta, 2011). El dosel superior del bosque alcanza los 40 metros de altura; es muy denso y semideciduo. De hecho, el 35\% de las especies pierde sus hojas durante la estación seca, lo que las hace el mayor número de especies deciduas del piso de baja altitud. Se sitúan sobre formas de origen estructural o de sedimentación aluvial, de topografía accidentada o moderada con serranías, valles angostos y gargantas con forma moderada plana a cóncavo o plano ondulado y suelos poco profundos. Este bosque está constituido por especies como espavel, ojoche, ceibo, guácimo colorado, peine de mico, palma real, coyol y especies secundarias como guarumo, capulín, marañón y nance (Porta, 2011).

-Bosque siempre verde estacional: El bosque siempre verde estacional de bajura está situado sobre formas de sedimentación aluvional (aluviones), con topografía moderada de plano ondulada a sistemas de colinas y cerros bajos e irregulares. Su topografía es accidentada, de serranías y valles angostos. Este tipo de bosque suele ser asociado con suelos arcillosos y pedregosos; latisoles pardos o pardo amarillentos. Además, presenta temperaturas medias anuales de 21 a $24{ }^{\circ} \mathrm{C}$ (Porta, 2011).

La vegetación tiene zonas sabanoides y de matorral, y zonas de agua con vegetación hidromórfica, sobre formas de sedimentación aluvial y materiales volcánicos. Las regiones que presentan este tipo de vegetación tienden a ser secas por efecto de su posición geográfica y orografía, manifiestas en la presencia de epífitas xerófilas. Presenta un dosel arbóreo con Cordia dentata, Quercus aata, Q. brenesi, Croton panamensis, Persea sp, Nectandra spp, Inga sp, Ardisia guianensis y Clusia spp., entre las que están: Clusia salvinii, Heliocarpus appendiculatus, Cecropia sp, Malpighia glabra, Terminalia sp, Chaeptoptelea mexicana, Ficus glabrata, Swetenia macrophylla (caoba), Sideroxylum capiri (tempisque), Astronium graveolens (ron ron), Platyismicium curuense (cachimbo) y Guaiacum sanctum (guayacán real).

La tabla 1 muestra las especies con los valores más altos del índice de valor de importancia, a pesar de que se encontró gran diversidad de especies, ya que se registraron 431 individuos en total; la dominancia, frecuencia y abundancia marcaron diferencias en cuanto al IVI en general. Ejemplo de lo anterior se puede apreciar con Swietenia macrophylla que, aún con baja abundancia, tiene una alta área basal, por lo que alcanza el 21,3\% del 
Revista Universidad en Diálogo • Vol. 7, N. ${ }^{\circ}$ 1, Enero-Junio 2017, pp. 101-122

ISSN 2215-2849 • EISSN: 2215-4752

DOI: http://dx.doi.org/10.15359/udre.7-1.6

IVI. En segundo lugar, se encuentra Bursera simarouba, la cual mostró la mayor cantidad de individuos (28) y fue encontrada en más cantidad de parcelas (19). Cabe agregar que entre las especies más representativas están Pseudobombax septenatum, Enterolobium cyclocarpum y Anacardiun excelsum, destacadas por presentar alta dominancia, pero cuentan con gran cantidad de individuos muestreados como otras especies. Lo anterior se debe a que son árboles de gran tamaño y buena cobertura de copa.

Tabla 1

Índice de valor de importancia, abundancia, dominancia y frecuencia relativa de las diez especies más representativas en la Reserva Biológica Lomas Barbudal, Bagaces, Guanacaste, Costa Rica

\begin{tabular}{lccccc}
\hline \multicolumn{1}{c}{ Especie } & Abun (\%) & Dom (\%) & Frec (\%) & I.V.I (Abs) & I.V.I (\%) \\
\hline Swietenia macrophylla & 4,176 & 12,730 & 4,360 & 21,267 & 7,089 \\
Bursera simarouba & 6,497 & 7,261 & 5,523 & 19,281 & 6,427 \\
astronium graveolens & 2,552 & 5,464 & 3,198 & 11,214 & 3,738 \\
Pseudobombax septenatum & 2,088 & 5,810 & 2,326 & 10,224 & 3,408 \\
Bursera graveolens & 3,944 & 2,076 & 3,488 & 9,509 & 3,170 \\
Bombacopsis quinata & 1,624 & 3,533 & 2,035 & 7,192 & 2,397 \\
Cochlospermum vitifolium & 2,552 & 1,474 & 2,907 & 6,933 & 2,311 \\
Sapranthus palanga & 2,320 & 1,828 & 2,616 & 6,764 & 2,255 \\
Enterolobium cyclocarpum & 1,160 & 4,094 & 1,453 & 6,708 & 2,236 \\
Anacardiun excelsum & 0,928 & 3,717 & 0,872 & 5,517 & 1,839 \\
\hline Subtotal & 27,842 & 47,988 & 28,779 & 104,609 & 34,870 \\
\hline Otras 77 sp. & 72,158 & 52,012 & 71,221 & 195,391 & 65,130 \\
\hline Total & $\mathbf{1 0 0}$ & $\mathbf{1 0 0}$ & $\mathbf{1 0 0}$ & $\mathbf{3 0 0}$ & $\mathbf{1 0 0}$ \\
\hline Nota: Elaborat & & & & &
\end{tabular}

Nota: Elaboración propia. 
En relación con índices de diversidad y con base en lo muestreado, se encontró que hasta el momento el sitio no presenta alta heterogeneidad biológica, dado que obtuvo un coeficiente de mezcla del 1:20,2, lo que indica que de cada veinte individuos muestreados es posible encontrar uno de diferente especie. Esto se debe a la gran cantidad de árboles por hectárea presentes en la zona, y no a la cantidad de especies encontradas. Además, el índice de Simpson mostró un valor de 1.86, por la alta dominancia que presentan ciertas especies con respecto a otras, pero es menos equitativo por su gran variedad, por lo que se puede considerar un sitio con gran diversidad. Con respecto al índice de Shannon, se obtuvo un valor de 4,23. Este último se basa en la probabilidad de encontrar un individuo dentro de la población, por lo que se considera un sitio diverso, pues los valores de este índice oscilan entre 1 y 5 , considerando diversos los valores mayores a 3 .

La abundancia de algunas especies y los rangos de distribución se determinan por las condiciones del terreno y por la interacción que entre ellas presentan.

El índice de similitud de Simpson (tabla 2) indica que entre las áreas muestreadas existen especies que coinciden en dos o tres tipos de bosque, lo que se relaciona con el índice de valor de importancia.

Tabla 2

Índices de similitud entre tipos de vegetación presentes en la Reserva Biológica Lomas Barbudal, Bagaces, Guanacaste, Costa Rica

\begin{tabular}{cccc}
\hline & B.S.V.E & B.S.B & B.S.DE \\
\hline B.S.V.E & $X$ & 0,421 & 0,433 \\
B.S.B & 0,421 & $X$ & 0,667 \\
B.S.DE & 0,433 & 0,667 & $X$ \\
\hline
\end{tabular}

Nota: Elaboración propia.

Los resultados mostrados en la figura 1 se obtuvieron según la evaluación silvícola: el 65\% (279) de los individuos reciben luz emergente, lo que indica que el bosque tiene buena entrada de luz para gran parte de las especies, debido a que solo un $1 \%$ de los individuos está cubierto. No obstante, se debe tomar en cuenta que la competencia de las especies por situarse en el dosel ha disminuido, además de que existen especies tolerantes a la sombra (figura 1). 


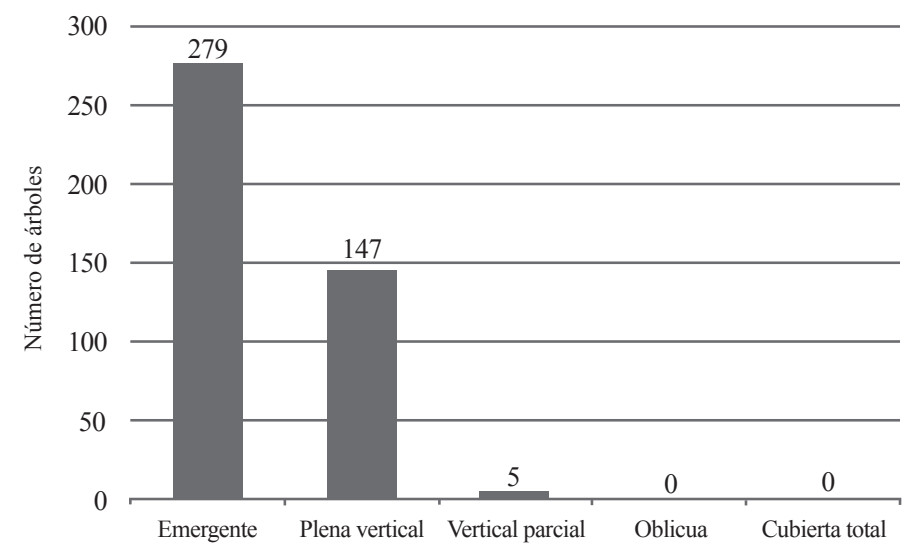

Figura 1. Grado de iluminación de las especies en RBLB, Bagaces, Guanacaste.

Nota: Elaboración propia.

E1 33\% de los individuos (143) presenta lianas en el fuste, 69 cuentan con gran incidencia de bejucos y enredaderas, 55 presentan bejucos y enredaderas en gran parte de la copa y 14 presentan la más alta infestación, dado que tienen tanto en el fuste como en copas, lo que implica alta competencia para obtener nutrientes entre los árboles y los bejucos o enredaderas.

Sin embargo, de los individuos muestreados, el 51\% (219) se encuentra libre de enredaderas o bejucos, lo que significa que el bosque, por las condiciones de incidencia de bejucos y enredaderas que presenta, es un bosque en el cual estas no se han manifestado aún como un problema grave que llegue a afectar al árbol, por lo que se destaca que la presencia de la luz puede jugar un papel importante en esta variable, lo cual resulta beneficioso. En este tipo de bosque, durante la época seca, los árboles botan sus hojas como mecanismo de supervivencia, lo que disminuye la cantidad de lianas (figura 2).

Otra característica encontrada es que el $52 \%$ de los individuos presenta fustes torcidos o deformados, o incluso muy torcidos o dañados; mientras que el $48 \%$ presenta buena forma del fuste, lo que indica que son árboles con potencial para ser utilizados como semilleros o como progenitores en programas de mejoramiento genético (figura 3 ). 


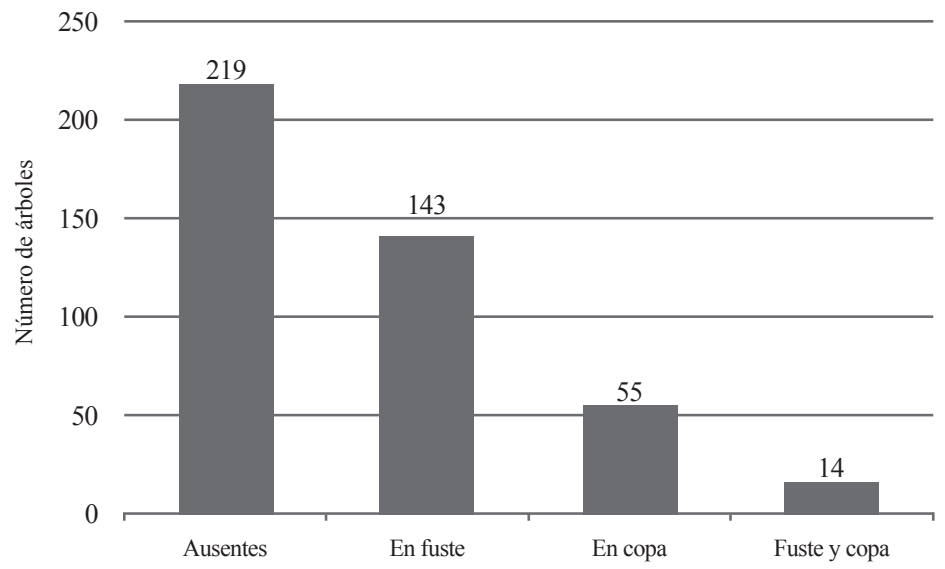

Figura 2. Incidencia de bejucos y enredaderas de las especies en RBLB, Bagaces, Guanacaste.

Nota: Elaboración propia.

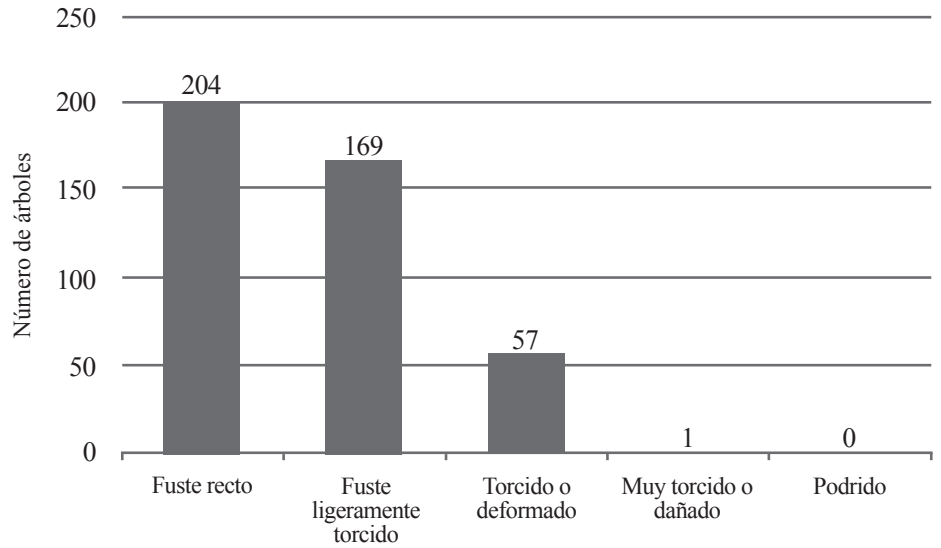

Figura 3. Calidad del fuste de las especies en RBLB, Bagaces, Guanacaste.

Nota: Elaboración propia.

De los individuos muestreados, solo 5 presentaron copa de mala forma, pues contaban con pocas ramas, 141 tienen copa completa o círculo completo y 223 lucen un círculo irregular (figura 4), lo que se asocia con el 
grado de iluminación y con la buena forma de los árboles, dado que muy pocos individuos cuentan con poca infestación de lianas y carecen de buen fuste. Esto resulta conveniente para un posible programa de extracción de semillas, pues indica que la población de árboles se encuentra en buen estado. Además, esto se puede relacionar con la edad de los individuos, debido a que son bosques relativamente jóvenes y en regeneración.

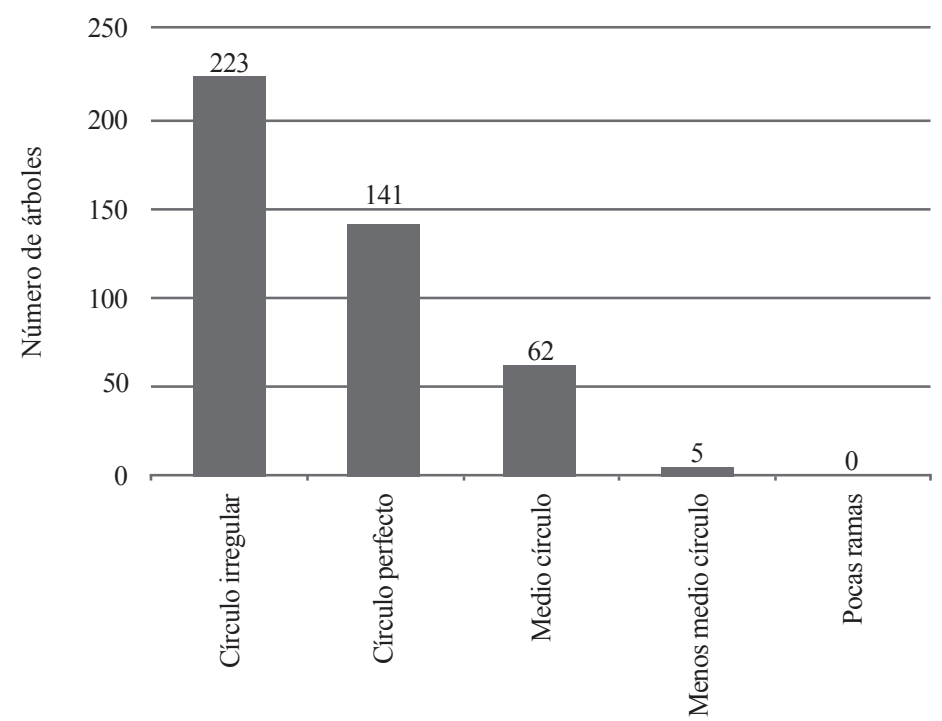

Figura 4. Forma de copa de las especies en RBLB, Bagaces, Guanacaste.

Nota: Elaboración propia.

Se obtuvo un volumen total de $594.23 \mathrm{~m}^{3}$ de madera, de los cuales 408.11 $\mathrm{m}^{3}(68 \%)$ corresponden a especies comerciales; entre estos $55.61 \mathrm{~m}^{3}$ de especies amenazadas de peligro de extinción y $117.89 \mathrm{~m}^{3}$ pertenecientes a Platymiscium parviflorum, Swietenia macrophylla y Dalbergia retusa, especies que cuentan con veda de aprovechamiento por Decreto Ejecutivo. De los 431 individuos registrados 41 cuentan con individuos con DAP superior a $60 \mathrm{~cm}$, que es el diámetro mínimo de corta establecido para la mayoría de especies, con un volumen total de $285.72 \mathrm{~m}^{3}$. Las principales especies que presentan volumen comercial son: Swietenia macrophylla, Bursera simarouba, Astronium graveolens, Bombacopsis quinata, Anacardiun excelsum, Enterolobium cyclocarpum y Ceiba pentandra. 
La RB Lomas Barbudal (figura 5) conecta directamente con el Corredor Biológico Rincón-Barbudal en la parte norte, el cual cuenta con una extensión de 29816 ha y recorre los distritos de Bagaces y Mogote, del cantón de Bagaces. Este, a su vez, conecta con el Parque Nacional Rincón de la Vieja. Además, en la parte sureste vincula con el Corredor Biológico Fila Nambiral, el cual tiene una extensión de 10999 ha que se extiende por la parte sur del distrito de Bagaces y que, también, enlaza con el Parque Nacional Palo Verde. Finalmente, RB Lomas Barbudal conecta directamente con este último en dos puntos en su parte sur, dado que cuenta con una extensión de 19800 ha y comparte con Lomas Barbudal gran diversidad en flora y fauna. Debido a esto, es una de las principales fuentes de intercambio genético entre especies con las que cuenta dicha reserva. Palo Verde, a su vez, vincula con los Corredores Biológicos Fila Nambiral, Paso del Mono Aullador y Chorotega, así como con la RVS Cipancí (ITCR, 2014).

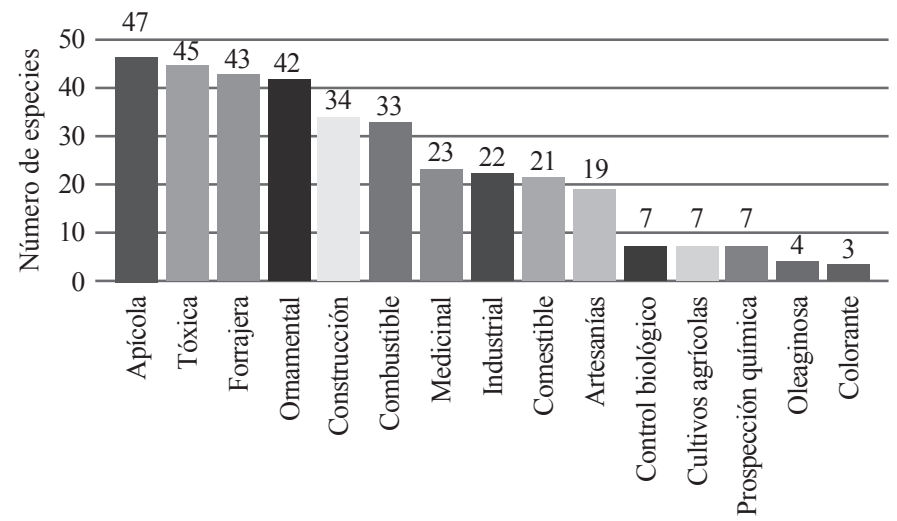

Figura 5. Riqueza de árboles encontrados según su uso.

Nota: Elaboración propia.

\section{Discusión}

Comparando lo observado en el campo con los resultados de Spittler (2002) sobre la dinámica de los bosques secos, se encuentran tres fases sucesionales en RB Lomas Barbudal, la primera fase arbustal abierto (o pastizal arbustivo): caracterizada por especies arbustivas como Acacia collinsii, Acacia farnesiana, etc. y especies pioneras como Gazuma ulmifolia y Cochlospermun vitifolium, entre otras, además de la presencia de algunas gramíneas. Esto principalmente en algunos sectores de bosque semideciduo de bajura. 
Revista Universidad en DiÁlogo • Vol. 7, N. ${ }^{\circ}$ 1, Enero-Junio 2017, pp. 101-122

ISSN 2215-2849 • EISSN: 2215-4752

DOI: http://dx.doi.org/10.15359/udre.7-1.6

Además se encuentra la cuarta fase bosque secundario intermedio, donde se da una paulatina reducción en importancia de las especies arbóreas pioneras, que poco a poco son reemplazadas por las especies heliófitas durables y en menor grado por las esciófitas; se pueden identificar dos estratos arbóreos, donde el estrato superior alcanza hasta los 15 metros. Y la quinta fase de bosque secundario tardío, cuyas características van semejando un bosque primario, donde las especies pioneras se tornan en oportunistas a la formación de algún claro, existe un dominio de las heliófilas durables pero las esciófitas van ganando cada vez más espacio y los estratos superiores rondan los 25 metros de altura.

Otro aspecto a considerar es que en la mayoría de la bibliografía consultada presentaba datos sobre especies que no aparecen en la R.B. Lomas Barbudal o se mezcló información de comunidades vegetales con las zonas de vida, considerándolas como elementos similares cuando no lo son. Este problema se suscita porque muchas veces la información ha pasado por varias modificaciones y no se evalúa su correspondencia con la realidad. En este caso ya se están presentando datos reales y actuales sobre cómo están distribuidas y cuáles especies hay en RB Lomas Barbudal.

Conviene señalar que las áreas con mayor similitud son el bosque semideciduo de bajura (BSB) y los bosques semideciduo y deciduo (BSDe) con un $66,7 \%$; las especies que más coinciden en ambos son: caraño (Bursera graveolens) con catorce individuos, seguido por caoba (Swietenia macrophylla) con trece individuos, además de ceiba (Pseudobombax septenatum), nanciguiste (Diospyrus salicifolia) y guaitil (Genipa americana) con más de cinco individuos en común cada una. El bosque siempre verde estacional (BSVE) presenta menor similitud con respecto a los otros dos sitios, pues comparte un $42 \%$ de especies con el BSB; de entre las más representativas son: espavel (Anacardiun excelsum), caraño y caoba. Con respecto al BSVE y el BS, existe un 43,3\% de similitud de especies como caraño, ceiba, zopilote (Sapranthus palanga) y aromo (Acacia farnesiana), pues son las que más individuos tienen en común.

Además, las especies con mayor incidencia entre los tres tipos de vegetación son: caoba, caraño y poro poro (Cochlospermum vitifolium). De estas especies, la caoba presenta mayor IVI, lo que se debe a su gran dominancia, aunque luego de considerar su distribución entre los tipos de vegetación, constituye una de las que más está presente. Esta situación es similar para el poro poro y caraño. Caso contrario sucede con el indio desnudo (Bursera simarouba), segunda especie con mayor IVI, aunque 
en su distribución por tipos de vegetación solo se encuentra en BSVE y BSB, sin embargo, su mayor incidencia se da en este último. También, esta especie es una de las que presenta mayor número de individuos, aunque su distribución se limita según las condiciones del terreno.

Estas diferencias se deben al número de árboles por parcela, además de las diferencias entre diámetros y alturas. También, otro factor que varía en menor grado los resultados es la densidad específica de las especies. Si se comparan estos resultados con otros obtenidos por estudios realizados, se puede observar que estos se encuentran entre un rango normal, ya que Ulate (2011) encontró en el bosque seco tropical valores de 161.33 t/ha para bosques primarios, en bosques secundarios $129.33 \mathrm{t} / \mathrm{ha}$ y $79.58 \mathrm{t} / \mathrm{ha}$ en bosques secundarios tardíos con más de veinte años de edad. Por su parte, Watson et al (2002) identificaron valores de 150.2; 138.1; y $149.9 \mathrm{t} / \mathrm{ha}$ en tres sitios de bosque tropical seco; asimismo estos autores indican que condiciones como evapotranspiración, temperatura, precipitación media de los sitios y factores edáficos pueden ocasionar variaciones en los datos de biomasa en cada sitio.

Se debe considerar la trascendencia de la función que tienen los árboles de RB Lomas Barbudal al captar el carbono atmosférico almacenado en esta área. Lo anterior debido a que los ciclos naturales de silvigénesis de los bosques se encuentran en constante movimiento en cuanto a la captura, almacenamiento y liberación de este elemento químico. Sin embargo, según las condiciones presentes en el bosque, puede que se encuentre en los topes de fijación de carbono, debido a que queda almacenado y no es devuelto al sistema tan constantemente como ocurriría en bosque bajo manejo o plantaciones forestales.

Como primer elemento de análisis, de las 86 especies forestales registradas, 47 han sido utilizadas o se podrían utilizar para practicar la apicultura, aunque no sea muy relevante en la región donde se ubica esta reserva biológica, pues la prioridad de los propietarios de las fincas de la zona es la ganadería y los cultivos de arroz y caña de azúcar. No obstante, esto último afectaría fuertemente a la apicultura por la cantidad de agroquímicos empleados. En contraste, la RBLB se caracteriza por ser una de las regiones con mayor cantidad de insectos en el plano nacional, por lo que es conocida como el Parque de los Insectos, por la gran variedad que resguarda. Cabe agregar que cuenta con registros de 230 especies de abejas y avispas únicas en el país (Serrano, 2012). Aunado a lo anterior, se encontró que 42 especies de las registradas son ornamentales o tienen el potencial para 
ello, principalmente por el colorido de sus flores. Lomas Barbudal es reconocida por sus "mega floraciones" (Porta, 2011), presentes entre los meses de noviembre y abril, cuando la mayoría de los árboles pierden sus hojas y se resalta más lo llamativo de sus flores, como, por ejemplo, el cortés negro y amarillo, roble sabana, saíno, cocobolo y vainillo.

También, se descubrió que 43 especies de las muestreadas son forrajeras, por lo que sus semillas podrían ser utilizadas para la siembra de árboles en fincas aledañas y, de este modo, se aprovecharían para brindar sombra y alimento al ganado. Estas especies son bien conocidas, incluso algunas son de rápida propagación, tales como el capulín, la guaba, el jícaro o el indio desnudo. Además, se descubrió que 23 especies producen frutos alimenticios para el ser humano, lo que puede ayudar a expandir el comercio y diversificar la producción en las fincas cercanas a la RBLB, por medio del establecimiento de sistemas agroforestales. De igual manera, se encontraron siete especies que ya han sido utilizadas para el control biológico, gracias a ciertos compuestos u olores que liberan de sus estructuras, por lo que dichas especies podrían ser estudiadas para crear productos derivados.

Existen 34 especies que se utilizan para la construcción, por lo que resulta evidente que Lomas Barbudal posee un gran banco para programas de mejoramiento genético. Al tomar en cuenta lo anterior, conviene agregar que a pesar de que los árboles presentes en la zona de estudio son de regeneración natural, poseen en su mayoría buena forma de fuste y copas.

También existen 22 especies para uso industrial, específicamente para la elaboración de productos altamente tecnificados (Poveda y Sánchez, 1999), de los cuales se derivan chicles o gomas de mascar, fósforos, resinas y tintes, entre otros. Con respecto a lo anterior, se encuentran tres especies que han sido comúnmente utilizadas para la producción de tintes, de las que se consiguen fácilmente los colorantes, tales como: mora (Maclura tinctoria), guaitil (Genipa americana) y nance (Byrsonima crassifolia) (Poveda y Sánchez, 1999).

En esta reserva existen cuatro especies oleaginosas, de estas se extraen aceites, ellas son: vainillo (Tecoma stans), aceituno (Simarouba glauca), roble encino (Quercus oleoides) y alcornoque (Licania arbórea). No obstante, de las especies registradas, 45 presentan elementos tóxicos, dañinos tanto para el ser humano como para ciertos animales. También tres especies presentan un alto potencial de contener dichas toxinas, razón por la cual se 
encuentran en estudio actualmente, dado que su prospección química presenta un alto potencial científico-tecnológico (Poveda y Sánchez, 1999). Algunos ejemplos de ellas son: Trichilia americana, Tabebuia impetiginosa y Senna atomaria. Asimismo, se encontraron 23 especies que han sido utilizadas con fines tradicionalmente medicinales, por ejemplo, malestares estomacales, dolores de articulaciones, dolencias dentales u otras.

Existe otro uso que se le da a siete de las especies registradas, así como en cultivos agrícolas en función de barreras rompe vientos, postes para cercas, generadoras de sombra y demás. De las 87 especies registradas, 33 tienen propiedades para ser utilizadas como combustible, leña o carbón, razón por la cual estas especies en temporada de incendios forestales deben ser las más vigiladas, debido a las condiciones del bosque y a lo rápido que se propaga el fuego en la zona. Por último, 19 especies han sido utilizadas para la elaboración de artesanías, ya sea por sus maderas o por sus frutos, como en el caso del jícaro (Crescentia alata) y el bala de cañón (Couroupita nicaraguarensis).

Con respecto al material genético resguardado en la Reserva Biológica Lomas Barbudal, se descubrió que, según una clasificación realizada por Poveda y Sánchez (1999) sobre la distribución de las especies de árboles y palmas del Pacífico Norte de Costa Rica, 45 son muy abundantes en la región, 25 son abundantes, 12 son escasas o con poblaciones muy reducidas y 4 se encuentran en alto riesgo de erosión genética, debido a su distancia entre individuos y los pocos ejemplares que de ellas existen. Estas especies son: caoba (Swietenia macrophylla), cristóbal (Platymiscium parviflorum), cocobolo (Dalbergia retusa) y bala de cañón (Couroupita nicaraguarensis), las cuales se ven altamente amenazadas por sus cotizadas maderas, además de que no existen plantaciones forestales de este mismo tipo que actualmente suplan los mercados, pues lo que hay son ensayos establecidos; aunque todavía no llegan a tener gran peso en el comercio forestal, como es el caso de las plantaciones de otras especies.

Otro de los componentes de protección de los recursos naturales en RBLB consiste en diez nacientes de agua permanentes, de las que se obtiene el agua que abastece la comunidad de San Ramón de Bagaces. También, existen las poblaciones de fauna: una de las más estudiadas son los monos cara blanca (Cebus capucinus), los cuales obtienen sus alimentos de los frutos y flores de 26 especies forestales registradas, así como de las hormigas presentes en el cornizuelo (Acacia collinsii) y el aromo (Acacia farnesiana) (Campos, 22 de junio, 2013, comunicación personal). 
Revista Universidad en DiÁlogo • Vol. 7, N. ${ }^{\circ}$ 1, Enero-Junio 2017, pp. 101-122

ISSN 2215-2849 • EISSN: 2215-4752

DOI: http://dx.doi.org/10.15359/udre.7-1.6

\section{Potencial de conectividad}

La conectividad entre áreas es la capacidad de un paisaje de mantener el movimiento de organismos, genes, materiales o energía, a pesar de que, por lo general, el concepto esté principalmente relacionado con el movimiento de especies (Mann y Plummer, 1995). El método para mantener ese flujo entre áreas silvestres protegidas se da por medio de los corredores biológicos (CB), con el fin de lograr la supervivencia de las especies y una constante provisión de servicios ecosistémicos a largo plazo entre áreas que comparten características similares en cuanto a flora y fauna. El Sistema Nacional de Áreas de Conservación (Sinac) cuenta con el Programa Nacional de Corredores Biológicos, el cual se establece en el país como producto de la iniciativa Corredor Biológico Mesoamericano, concebida y organizada para brindar bienes y servicios ambientales a la sociedad mesoamericana y mundial. Esta procura la concertación social para promover la conservación de la biodiversidad, además del uso sostenible de los recursos naturales, y así contribuir a mejorar la calidad de vida de quienes habitan la región (Sinac, 2009).

Gracias al espacio en el que se ubica la Reserva Biológica Lomas Barbudal se le facilita contar con un flujo de intercambio genético directo con el Parque Nacional Palo Verde, por lo que tanto las condiciones de polinización como la dispersión de semillas se ven favorecidas al compararlas con otras áreas protegidas que quedan "aisladas" de distintas zonas de conservación. Además, con el establecimiento del Programa Nacional de Corredores Biológicos se establece una herramienta que demuestra el esfuerzo por conservar la biodiversidad y propiciar la producción de bienes y servicios amigables con la naturaleza, que ayuden a mejorar sus condiciones socioeconómicas (Sinac, 2009). Conviene recordar que el establecimiento de zonas que conecten las diferentes áreas protegidas del país beneficia la producción y conservación de diversos servicios ecosistémicos que, eventualmente, pueden promulgar más incentivos para el resguardo y aprovechamiento del recurso forestal del país.

Con respecto al flujo de intercambio genético generado por medio de la conectividad entre áreas, cabe decir que promulga el enriquecimiento de la flora y fauna. En el caso particular de RB Lomas Barbudal, esto puede ayudar a aquellas especies forestales que corren el riesgo de erosión genética o están en peligro de extinción, tales como: caoba (Swietenia macrophylla), cristóbal (Platymiscium parviflorum), cocobolo (Dalbergia retusa) y bala de cañón (Couroupita nicaraguarensis). 


\section{Conclusiones}

En el muestreo realizado se pudo observar la manera en que la frecuencia de especies, la dominancia y la abundancia tienen alta influencia en los valores del índice de valor de importancia (IVI); en este caso las especies con mayores valores encontrados fueron: Swietenia macrophylla, Bursera simarouba, Pseudobombax septenatum, Enterolobium cyclocarpum y Anacardiun excelsum, lo que hace indicar que este bosque ya se encuentra en un estado sucesional de bosque secundario tardío (Spittler, 2002), pues si se compara con otros estudios en diferentes regiones de bosque seco, se encuentra por ejemplo que una de las especies con mayor valor de IVI es Guazuma ulmifolia, además de otras especies como Glicicidia sepium y Tabebuia ochraceae, entre otras, por lo que, como lo mencionan Hernández et al. (2002), se da una tendencia a cambiar la composición del bosque conforme aumenta la edad.

En la forma de los árboles encontrados, la mayoría presenta buena forma de fuste y copa, a pesar de que crecen de manera natural. Otra ventaja que presenta la región es que existe buena entrada de luz a los doseles inferiores, lo que favorece los individuos juveniles.

También, por las observaciones realizadas en diferentes épocas, la incidencia de enredaderas o bejucos, las anuales, se ve afectada por las condiciones climáticas, dado que en época seca aquellos formados en la estación lluviosa se secan o caen por la pérdida de hojas de los árboles.

De las especies registradas se obtuvo un volumen de $594.23 \mathrm{~m}^{3}$, de los cuales $285.72 \mathrm{~m}^{3}$ es volumen comercial de especies con diámetro mayor a $60 \mathrm{~cm}$; hay tres especies que cuentan con veda por Decreto Ejecutivo, por lo que un $31 \%$ del volumen comercial no podría cortarse, en el caso hipotético de un aprovechamiento.

Uno de los servicios ecosistémicos encontrados es la captación de biomasa de carbono. Se obtuvo valores de biomasa aérea que van desde 5.23 y $572.14 \mathrm{t} /$ ha en las parcelas registradas, con un valor promedio de 186.69 $\mathrm{t} / \mathrm{ha}$, estas diferencias se dan por el número y el tamaño de los individuos dentro de las parcelas; estos valores son similares a los obtenidos por Ulate (2011), quien encontró un promedio de 161.33 t/ha en bosque primario y 129.33 t/ha en bosque secundario en bosques secos del país, además se acercan a los valores obtenidos por Watson et al. (2002) en diferentes áreas de bosque seco. 
La diversidad de especies presentes en RB Lomas Barbudal muestra una rica fuente de material genético, pues de cada especie es posible encontrar algún uso comercial u ornamental, además de que existe un alto valor de productos no maderables que pueden ayudar en la economía de la región, como por ejemplo las especies con potencial apícola. Además, existen especies por analizar para obtener derivados medicinales.

Finalmente, pero no menos importante, la ubicación de la zona de estudio; sin duda, favorece el programa de Corredor Biológico Mesoamericano, en vista de que posee un banco genético diverso, además de que conserva especímenes en extinción o con pocas poblaciones. Esto facilita el intercambio genético con otros sectores, principalmente con el PN Palo Verde, que es el más cercano y posee condiciones muy similares, por lo que comparten variadas especies.

\section{Referencias}

Andrade, A. (2007). Aplicación del enfoque ecosistémico en Latinoaméri$c a$. Bogotá, Colombia: CEM - UICN.

Cascante, A., Estrada, A. (2000). Composición florística y estructura de un bosque húmedo premontano en el Valle Central de Costa Rica. Revista de Biología Tropical, $n^{\circ} 49$ (1 $1^{\text {a }}$. Ed.). Museo Nacional de Costa Rica, San José. C.R. 13 p.

Centro Agronómico Tropical de Investigación y Enseñanza (CATIE). (2002). Inventarios forestales para bosques latifoliados en América Central. (Eds. Lorena Orozco y Cecilia Brumér). Manual técnico / CATIE; $n^{\circ}$. 50, Turrialba, Costa Rica: CATIE.

Chave, J., Andalo, C., Brown, S., Cairns, M., Chambers, J., Eamus, D., Folster, H., Fromard, F., Higuchi, N., Kira, T., Lescure, J., Nelson, B., Ogawa, H., Puig, H., Riera, B., Yamakura, T. (2005). Tree allometry anda improved estimation of carbón stocks and balance in tropical forests [Alometría arbórea y una mejor estimación de las reservas de carbón y el equilibrio en los bosques tropicales]. Ecosystem ecology. Recuperado de http://chave.ups-tlse.fr/chave/ chave-oecologia05.pdf

Curtis, J. T. y McIntoch, R. P. (1950). Diversity and distribution of terricolous lichens as indicator of habitat heterogeneity and grazing induced trampling in a temperate-alpine shrub and meadow. Academiaedu. Recuperado de http://www.academia.edu/1077402/ 
Diversity_and_distribution_of_terricolous_lichens_as_indicator_ of_habitat heterogeneity_and_grazing_induced_trampling_in_a temperate-alpine_shrub_and_meadow

Doblado, L. (2011). Identificación y caracterización de tipos de bosque y su relación con variables ambientales, en un paisaje fragmentado al norte de Honduras (tesis de maestría). Recuperado de http://orton. catie.ac.cr/repdoc/A7430e/A7430e.pdf

González, E. (2002). Composición y dinámica de diferentes estados sucesionales en el bosque seco tropical del Parque Nacional Palo Verde Costa Rica. Ecosistemas forestales de bosque seco tropical: investigaciones y resultados en Mesoamérica (pp. 146-152). Heredia, Costa Rica: UNA, INISEFOR.

Hernández, V., Quesada, R. y González, E. (2002). Cronosecuencia del bosque seco secundario tropical en el Parque Nacional Palo Verde, Bagaces, Costa Rica. Ecosistemas forestales de bosque seco tropical: investigaciones y resultados en Mesoamérica (pp. 111-118). Heredia, Costa Rica: UNA, INISEFOR.

Instituto Tecnológico de Costa Rica (ITCR). (2014). Atlas digital de Costa Rica. Escuela de Ingeniería Forestal, Laboratorio de Información Geográfica. Cartago, Costa Rica: ITCR.

Mann, C. C. y Plummer, M. L. (1995). Are wildlife corridors the righ path? Science, 270, 1428-1430. Recuperado de http://science.sciencemag. org/content/270/5241/1428

Murrieta, E. (2006). Caracterización de cobertura vegetal y propuesta de una red de conectividad ecológica en el Corredor Biológico Volcánica Central-Talamanca (tesis de maestría). Recuperado de http:// www.ots.ac.cr/rdmcnfs/datasets/biblioteca/pdfs/nbina-7666.pdf

Porta, J. (2011). Reserva Biológica Lomas Barbudal. Ecosistemas de Costa Rica. Blog de la Biodiversidad de Costa Rica. Recuperado de http://ecosistemasdecostarica.blogspot.com.es/2012/11/reserva-biologica-lomas-de-bardubal.html

Poveda, L. J. y Sánchez, P. (1999). Árboles y palmas del Pacifico Norte de Costa Rica (claves dendrológicas). San José, Costa Rica: Guayacán.

Ramos, Z. (2004). Estructura y composición de un paisaje boscoso fragmentado: herramienta para el diseño de estrategias de conservación de la biodiversidad (tesis de maestría). Recuperado de http:// orton.catie.ac.cr/repdoc/A0303e/A0303e.pdf 
Ulate. C. A. (2011). Análisis y comparación de la biomasa aérea de la cobertura forestal según zona de vida y tipo de bosque para Costa Rica (tesis de licenciatura). Recuperado de http://orton.catie.ac.cr/ repdoc/A11141e/A11141e.pdf

Serrano, J. (2012). Reserva Biológica Lomas Barbudal. MINAET, SINAC, ACA-T. Tilarán. Costa Rica: RBLM.

Sistema Nacional de Áreas de Conservación (Sinac). (2009). Plan Estratégico del Programa Nacional de Corredores Biológicos de Costa Rica para el Quinquenio 2009-2014. Recuperado de http://www. sinac.go.cr/corredoresbiologicos/documentacion/plan_estrategico_ pncb.pdf

Spittler, P. (2002). Dinámica de los bosques secundarios secos en la Región Chorotega, Costa Rica. Ecosistemas forestales de bosque seco tropical: investigaciones y resultados en Mesoamérica (pp. 163-174). Heredia, Costa Rica: UNA, INISEFOR.

Watson, V., Kauffman, B., Hughes, R. F., Tosi, J. A., Jobse, J., Hughes, A. y Kauffman, D. (2002). Contenido de carbón en el bosque seco tropical. Ecosistemas forestales de bosque seco tropical: investigaciones y resultados en Mesoamérica (pp. 111-118). Heredia, Costa Rica: UNA, INISEFOR. 